\title{
La situación laboral de las personas con discapacidad en Colombia desde un enfoque local
}

\author{
VELANDia Campos, JoAn SEBAstian \\ Pontificia Universidad Javeriana Cali (Colombia) \\ Correo electrónico: sebastian.velandia@javerianacali.edu.co \\ CAstillo Caicedo, MARIBEL \\ Pontificia Universidad Javeriana Cali (Colombia) \\ Correo electrónico: mabelcas@javerianacali.edu.co \\ MORA RODRÍGUEZ, JHON JAMES \\ Universidad ICESI (Colombia) \\ Correo electrónico: jjmora@icesi.edu.co
}

\section{RESUMEN}

Este artículo analiza las dinámicas locales del mercado laboral para población con discapacidad (PCD) en cinco importantes ciudades de Colombia. A partir de los datos obtenidos del Registro para la Localización y Caracterización de Personas con Discapacidad (2018) y del Servicio Público de Empleo (2018) se estimaron las tasas locales de ocupación, desempleo, informalidad e ingresos de la PCD y además se construyó una función de emparejamiento laboral para determinar las posibilidades de acceso que brinda el mercado laboral a la PCD. Los resultados muestran tasas municipales de desempleo que oscilan entre el $17,0 \%$ y el $41,0 \%$, tasas de ocupación sin contrato entre el $69,0 \%$ y el $81,2 \%$, coberturas en capacitación para el trabajo de máximo 6,8\%; así como una función de emparejamiento con asimetrías con respecto a las probabilidades de encontrar trabajo $(26,1 \%)$ y de ocupar vacantes para la PCD $(73,9 \%)$. Finalmente, se revisaron los avances de iniciativas locales de empleo inclusivo para PCD que pueden considerarse como referencia de buenas prácticas para reducir las asimetrías entre la demanda y la oferta de trabajo.

Palabras clave: discapacidad, mercado laboral, función de emparejamiento, datos de panel. Clasificación JEL: J14; 015; J08; R58.

MSC2010: 91B40; 97b20; 91G70.

Artículo recibido el 1 de noviembre de 2019 y aceptado el 30 de marzo de 2020 


\title{
The employment situation of disabled people in Colombia from a local approach
}

\begin{abstract}
This article analyzes the local dynamics of the labor market for the population with disabilities (PCD) in five main cities of Colombia. Based on data from the Register for the Location and Characterization of Persons with Disabilities (2018), the rates of employment, unemployment, informality and income are analyzed, among other indicators of the PCD. Also, from the data of the Public Employment Service (2018), a labor match was estimate in order to determine the possibilities of access that the labor market offers to the PCD. Our results show the unemployment rates that oscillate between $17,0 \%$ and $41,0 \%$, the information rates between $69,0 \%$ and $81,2 \%$, coverage in the training for work of maximum $6,8 \%$; as well as a matching function with asymmetries with respect to the probabilities of finding work $(26,1 \%)$ and filling vacancies for PCD $(73,9 \%)$. Finally, we reviewed the progress of various local inclusive employment initiatives for PCD that can be considered as a reference of good practices to reduce asymmetries between demand and labor supply.
\end{abstract}

Keywords: disability, labor markets, inclusive employment, matching function.

JEL classification: J14; 015; J08; R58.

MSC2010: 91B40; 97b20; 91G70.

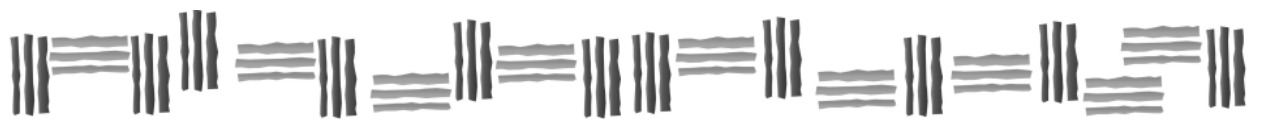




\section{Introducción.}

La población con discapacidad se enfrenta de forma general a distintas problemáticas de inserción laboral que tienen como efecto una situación desigual con respecto al resto de la población activa y ocupada al presentar tasas de actividad, ocupación y probabilidades de empleo inferiores (Cabrero et al., 2009). Además, en varias investigaciones (Velandia et al., 2018; Espinosa, 2016; Correa \& Castro, 2016; Cruz \& Hernández, 2010; Hernández \& Hernández, 2005) se ha aportado evidencia científica para entender el contexto de las distintas problemáticas a las que debe enfrentarse la Población con Discapacidad (en adelante, PCD) en Colombia, así como evidencia suficiente sobre las disparidades que coexisten entre tal colectivo y el resto de la población.

Además de tales avances, los objetivos a nivel de país han apuntado a hacer un seguimiento socioeconómico nacional de la PCD a nivel municipal, departamental, distrital y nacional, que permita precisar sus necesidades contractuales en los distintos niveles administrativos para lograr un alcance más efectivo en la planificación y ejecución de acciones, programas y proyectos. Para tal fin, desde el Ministerio de Salud y Protección Social se ha avanzado con una estrategia de recogida de datos a través del Registro para la Localización y Caracterización de Personas con Discapacidad (en adelante, RLCPD) que para el 2018 reúne información de 1.418 .065 personas con discapacidad equivalentes al 2,8\% de la población colombiana (Ministerio de Salud y Protección Social, 2018a; DANE, 2018).

No obstante, tales avances de seguimiento aún no logran alcanzar el 6,3\% de la población total identificada como PCD por el censo del año 2005 y peor aún, en los distintos niveles administrativos continúa haciendo falta contar con indicadores laborales de base local para la PCD que permitan en términos técnicos cumplir con tales objetivos e instrumentalizar un adecuado seguimiento, tratamiento y evaluación de las medidas pactadas para responder a las necesidades de la PCD.

Es por esto que, comprendiendo que es en el ámbito local donde surgen las interacciones directas entre los distintos agentes que intervienen en el mercado de trabajo como lo son la orientación, la intermediación, la contratación laboral y demás relaciones, este artículo busca contribuir a las prioridades de la Agenda de Investigación en Discapacidad para Colombia (Cruz et al., 2017); al aportar la construcción de un análisis local del mercado de trabajo para PCD, logrado a partir de una aproximación a la situación actual del mercado y de sus desequilibrios, así como de sus respectivos casos locales de éxito en inserción laboral. La Agenda de Investigación en Discapacidad para Colombia prioriza, en el área "Productividad, trabajo, empleo y emprendimiento", los siguientes temas y subtemas: 1. Análisis del mercado laboral (desempleo, informalidad, precariedad laboral, evaluación y análisis econométrico del mercado laboral) 2. Análisis de la política de acceso al empleo (políticas locales, empleo público y privado, educación para el trabajo, ley de cuotas) y 3 . Análisis del Bienestar laboral (empleo con apoyo, promoción dentro del empleo, movilidad en el empleo, análisis de riesgos).

Los datos del presente artículo provienen del Sistema Integral de Información de la Protección Social (Ministerio de Salud y Protección Social, 2018a) entre los que un 69,0\% de la PCD no se encuentran impedidos para trabajar pese a su discapacidad y, por tanto, representan específicamente la población objetivo del estudio. También se usaron datos de registro, orientación, colocación y vacantes para PCD del Servicio Público de Empleo en el año 2018. Específicamente la unidad de análisis local es definida como las dinámicas que presentan cinco principales cabeceras municipales de Colombia: Bogotá, Medellín, Cali, Bucaramanga y Barranquilla, que coinciden en estricto orden con las ciudades con mayor representatividad de la población en estudio concentrando un total de $33,2 \%$ de la PCD de Colombia (Ministerio de Salud y Protección Social, 2018a).

El artículo se organiza de la siguiente manera. En la sección dos se realiza una caracterización local de las condiciones laborales de la PCD. En la sección tres se presenta un modelo de emparejamiento con sus resultados. En la sección cuatro se muestran casos locales exitosos sobre inserción laboral y, finalmente, se presentan las conclusiones. 


\section{Caracterización del mercado laboral de la PCD en Colombia.}

Diferentes autores (Velandia et al., 2018; Espinosa, 2016; Correa \& Castro, 2016; Cruz \& Hernández, 2010; Hernández \& Hernández, 2005) han aportado evidencia científica para entender el contexto de las distintas problemáticas a las que debe enfrentarse la $\mathrm{PCD}$, así como evidencia suficiente sobre las disparidades que coexisten entre tal colectivo y el resto de la población.

Distintas medidas dirigidas a la PCD han surgido a partir de iniciativas locales transformadas en Políticas Activas de Empleo (PAE) que han centrado sus objetivos en la inclusión sociolaboral, la generación de calificaciones y competencias laborales, la promoción del autoempleo, así como el desarrollo del emprendimiento asociativo (Fundación Demuca, 2011) y, por lo general, agrupan medidas como la creación de servicios públicos de empleo, trabajo protegido y cuotas de empleo, subvenciones al empleo con contrato indefinido, empleo con apoyo y/o adaptado, programas de rehabilitación, medidas de adaptación al trabajo, y cursos de formación ocupacional para parados y de formación continua para ocupados (Alburquerque, 2004; Cabrero et al., 2009).

No obstante, además de asegurar una convergencia productiva, garantizar la protección social, generar cambios institucionales adecuados y lograr un crecimiento económico sostenido con equidad (Infante \& Sunkel, 2009), el desarrollo inclusivo debe procurar la integración social de los distintos ciudadanos, así como valorar y generar las condiciones laborales, productivas y sociales adecuadas para aprovechar el papel que cada sujeto puede ejercer en el proceso de desarrollo una vez acceda a las distintas oportunidades (Berman-Bieler, 2007; Infante \& Sunkel, 2009).

Los resultados de lograr las condiciones anteriores se traducen, en términos económicos, en la reducción de los costos asociados al no aprovechamiento de los posibles aportes de capital humano del grupo de PCD y de los costos de oportunidad en que incurren sus hogares, esto dado que dentro de las dinámicas de la PCD no sólo se discrimina al sujeto en cuestión sino también a los hogares al que pertenece. Específicamente, los costos sobre la producción y los ingresos inducido por la falta de inclusión de la discapacidad alcanzan entre el 5,3\% y el 6,9\% del PIB mundial (Metts, 2004), mientras que los hogares con PCD deben enfrentarse a una mayor dependencia familiar, una inferior capacidad de generación de ahorro y a elevados costes directos e indirectos que pueden representar un $25 \%$ más de los gastos corrientes (Jiménez \& Huete, 2011; Parckar, 2008).

La información aquí utilizada proviene del Sistema Integral de Información de la Protección Social (SISPRO) del Ministerio de Salud y Protección Social de Colombia. A través de éste se recolectan desde el año 2002 datos de las personas con discapacidad con el fin de localizarlas y caracterizarlas en los diferentes niveles administrativos del país y teniendo esto en cuenta definir medidas específicas de política pública. Los datos son registrados en el Sistema de Gestión de Datos (SGD) o Bodega de Datos de SISPRO por 2.045 Unidades Generadoras de Datos (UGD) comprendidas por las instituciones, entidades y organizaciones de carácter público o privado que realizan atención, intervención o apoyo a la PCD en todo el país y son actualizados, en su caso, gracias a la interoperabilidad con las demás fuentes de información del sistema integral SISPRO (Ministerio de Salud y Protección Social, 2018a). Éste es un registro de carácter administrativo y voluntario, por lo que los datos obtenidos dan cuenta de la información que las PCD brindaron voluntariamente al momento de diligenciar o actualizar su información y por ello podrían presentar sesgo de selección. No obstante, es el principal sistema de información en materia de discapacidad de Colombia ya que es el más completo al registrar capítulos asociados con los niveles persona, hogar, localización, caracterización y origen de la discapacidad, salud, educación, participación social, y trabajo (Ministerio de Salud y Protección Social, 2014, 2018a) y el de mayor tamaño muestral al registrar 1.418.065 personas, por lo que el RLCPD es la fuente más viable para el análisis laboral de tal población.

La información del 2018 muestra que el 34,2\% de la PCD se encuentra impedida permanentemente para trabajar debido a su discapacidad y entre estos un $89,8 \%$ no reciben pensión por discapacidad. Del lado opuesto, entre quienes no se encuentran impedidos para trabajar únicamente el $11,8 \%$ respondieron estar trabajando en el momento del registro (11,8\% de la muestra), no obstante, 
con una situación laboral crítica, ya que entre estos el $82,1 \%$ trabajan sin ningún tipo de contrato laboral, lo que agrava las posibilidades de formalidad del empleo, que alcanza tasas tan críticas como solo un $1,7 \%$ de personas con pensión laboral.

En la Tabla 1, la PCD en edad para trabajar fue acotada por la PCD con edades comprendidas entre los 12 y 99 años, según la edad mínima de trabajo en Colombia, mientras que la PCD económicamente activa se acotó por el grupo en edad de trabajo cuya principal actividad en los últimos 6 meses fue trabajar o buscar trabajo.

A nivel local, Barranquilla es la ciudad que presenta la mayor tasa de desempleo de PCD con un $41,0 \%$, seguido por Cali $(38,9 \%)$ y Medellín $(31,1 \%)$. A su vez estas tres ciudades presentan un nivel de desempleo muy por encima del promedio obtenido a nivel de país, lo que deja en evidencia problemas estructurales de desempleo muy graves en los mercados locales de trabajo. No obstante, Barranquilla y Medellín difieren de Cali al presentar en el grupo los más altos niveles de desempleo de su PCD económicamente activa, acompañado de las peores tasas de ocupación para su PCD.

Cabe observar, que Bucaramanga y Bogotá son las únicas ciudades del grupo que sitúan una tasa de desempleo para PCD por debajo de la estimación nacional, alcanzando unos niveles relativamente aceptables de $17,0 \%$ y $18,1 \%$ respectivamente. No obstante, pese a que estas dos ciudades también lideran en relación con sus niveles de ocupación con tasas de $15,1 \%$ y $13,7 \%$ respectivamente, tales niveles siguen siendo muy bajos, incluso aunque se sitúen levemente por encima del promedio nacional, dejando por tanto evidencias de niveles de ocupación altamente críticos para todo el grupo de ciudades (Tabla 1).

Tabla 1. Indicadores locales de trabajo para PCD (2018).

\begin{tabular}{|l|cccccc|}
\hline & BOG & MED & CAL & BUC & BAR & COL \\
\hline PCD-ET: PCD en Edad para Trabajar* & 273.899 & 64.349 & 62.909 & 22.630 & 19.975 & 1.325 .610 \\
PCD-EA: PCD Económicamente Activa ${ }^{* *}$ & 45.878 & 7.217 & 13.602 & 4.106 & 2.964 & 223.441 \\
TD: Tasa de Desempleo & $18,1 \%$ & $31,1 \%$ & $38,9 \%$ & $17,0 \%$ & $41,0 \%$ & $25,7 \%$ \\
TO: Tasa de Ocupación & $13,7 \%$ & $7,7 \%$ & $13,2 \%$ & $15,1 \%$ & $8,8 \%$ & $12,5 \%$ \\
\hline
\end{tabular}

Fuente: Elaboración y estimaciones propias con base en RLCPD (Ministerio de Salud y Protección Social, 2018a).

Las mejores tasas en desempleo y ocupación que presenta la ciudad de Bucaramanga, no pueden evaluarse de forma favorable sin considerar la calidad del empleo otorgado al colectivo en estudio. Tales condiciones pueden analizarse considerando los tipos de contratos que presenta el grupo de trabajadores de PCD y que, en su caso, mejoran las probabilidades de formalización, acceso a la seguridad social y calidad del empleo. No obstante, se encontró que el $82,5 \%$ de los ocupados en Bucaramanga trabajan sin ningún tipo de contrato a término fijo o indefinido, lo que representa otra de las graves problemáticas laborales de la PCD a nivel local.

Por su parte, la ciudad de Bogotá que presentó la segunda tasa de desempleo más baja $(18,1 \%)$ y el nivel de ocupación más alto $(13,7 \%)$, presenta el mayor nivel de ocupados con contrato de trabajo al alcanzar una tasa de $29,1 \%$. Así mismo, la capital colombiana presenta el mayor porcentaje de trabajadores con contratos a término indefinido cuyo tipo suele estar cubierto por empleos con garantías de los derechos laborales. Sin embargo, todas las ciudades presentan cifras muy altas de ocupados sin ningún contrato laboral que oscilan entre el $69 \%$ y $81,2 \%$ de PCD, dejando expuesta la urgente necesidad de generar medidas que incentiven la formalización del empleo en términos de contrato y, en consecuencia, en términos de protección de la seguridad social (Tabla 2). 
Tabla 2. Tipo de contrato.

\begin{tabular}{|l|cccccccccccccc|}
\hline & \multicolumn{10}{|c|}{ PCD trabajando en los últimos 6 meses. } \\
& BOG & \% & MED & \% & CALI & \% & BUC & \% & BAR & \% & COL & \% \\
\hline Término fijo & 3.557 & 9,4 & 612 & 12,3 & 730 & 8,6 & 319 & 9,3 & 183 & 10,4 & 11.941 & 7,1 \\
Término indefinido & 7.490 & 19,8 & 778 & 15,6 & 1.044 & 12,4 & 284 & 8,3 & 137 & 7,8 & 18.133 & 10,8 \\
No aplica & 1 & 0,0 & 3 & 0,1 & 1 & 0,0 & 3 & 0,1 & N/A & 0,0 & 58 & 0,0 \\
No definido & 704 & 1,9 & 11 & 0,2 & 278 & 3,3 & 41 & 1,2 & 17 & 1,0 & 4.276 & 2,5 \\
Sin contrato & 26.164 & 69,0 & 3.579 & 71,8 & 6.395 & 75,7 & 2.793 & 81,2 & 1.419 & 80,8 & 133.321 & 79,5 \\
\hline Total Muestra & $\mathbf{3 7 . 9 1 6}$ & & $\mathbf{4 . 9 8 3}$ & & $\mathbf{8 . 4 4 8}$ & & $\mathbf{3 . 4 4 0}$ & $\mathbf{1 . 7 5 6}$ & $\mathbf{1 6 7 . 7 2 9}$ \\
\hline
\end{tabular}

Fuente: Elaboración propia con base en RLCPD (Ministerio de Salud y Protección Social, 2018).

$\mathrm{Al}$ analizar el tipo de ocupación laboral, las participaciones de trabajadores por cuenta propia son también considerablemente altas para todo el grupo de ciudades y son especialmente superiores en comparación con el promedio del país. Sin embargo, hace faltan variables locales que expongan si tal modalidad corresponde a empleo autogenerado de calidad, como por ejemplo por iniciativa de emprendimiento, o si hace parte de la economía informal.

Para este caso, Barranquilla, que anteriormente presentó la peor situación en desempleo y ocupación, presenta en este caso un repunte de $60,7 \%$ de PCD trabajando por cuenta propia. Por otra parte, en relación al empleo de origen público destaca el creado por la institucionalidad de Bogotá con un 7,4\% de empleados del gobierno; además la evidencia apunta a comprobar que las instituciones públicas de las ciudades en estudio están cumplimiento con las metas de cuotas de empleo que para el año 2019 oscilan entre $0,5 \%$ y $2 \%$ de trabajadores con discapacidad según el tamaño de la institución (Ministerio de Trabajo, 2017a); Bogotá también presenta la menor tasa local de trabajadores familiares sin remuneración (Tabla 3).

Tabla 3. Tipo de ocupación.

\begin{tabular}{|l|cccccc|}
\hline & \multicolumn{7}{|c|}{$\%$ de PCD } \\
\cline { 2 - 7 } & BOG & MED & CAL & BUC & BAR & COL \\
\hline Por cuenta propia & 45,6 & 50,1 & 45,7 & 44,8 & 60,7 & 40,1 \\
Empleado particular & 30,3 & 27,5 & 29,8 & 36,3 & 24,2 & 23,3 \\
Jornalero o peón & 3,3 & 9,9 & 4,2 & 3,8 & 2,7 & 16,9 \\
Empleado(a) doméstico(a) & 8,3 & 4,8 & 10,4 & 6,6 & 5,0 & 7,1 \\
Empleado del gobierno & 7,4 & 3,9 & 5,1 & 3,7 & 3,8 & 5,7 \\
Trabajador familiar sin remuneración & 1,0 & 1,7 & 2,4 & 1,4 & 2,4 & 4,0 \\
Patrón o empleador & 4,1 & 2,2 & 2,4 & 3,4 & 1,3 & 2,8 \\
\hline Total Muestra & 37.142 & 4.967 & 8.164 & 3.405 & 1.730 & 163.929 \\
\hline
\end{tabular}

Fuente: Elaboración propia con base en RLCPD (Ministerio de Salud y Protección Social, 2018a).

En cuanto a las actividades económicas de ocupación, destaca la participación en el sector de servicios con un $26,4 \%$ de los trabajadores y en menor medida el trabajo del sector industrial que alcanzan únicamente una tasa de 6,1\%; además una quinta parte de la PCD de Colombia está ubicada laboralmente en el sector agropecuario. 
En el grupo se cumple que, a excepción de Barranquilla, las ciudades repuntan con la generación de empleo local en actividades del sector servicios con participaciones superiores a la media de Colombia. Por otra parte, Bucaramanga vuelve a diferenciarse al obtener las participaciones más altas de PCD tanto en el sector industrial $(15,6 \%)$ como en el sector agropecuario $(3,9 \%)$, acompañado a su vez de una importante participación en los servicios (40,5\%) que solo es superada por la ciudad de Bogotá, lo que refleja la capacidad estructural de su mercado local de trabajo para avanzar en la equiparación sectorial del empleo inclusivo.

Barranquilla por su parte, es la ciudad con menor inserción laboral de PCD en la industria con una participación de apenas $4,7 \%$, que además se sitúa muy por debajo del promedio para el país; hecho que resulta paradójico si se considera que la ciudad es oficialmente catalogada como "Distrito Especial, Industrial y Portuario de Barranquilla" por lo que su agenda pública local necesita urgente crear medidas que alienten el empleo inclusivo en la industria (Gráfico 1).

Gráfico 1. PCD por Actividad Económica de Trabajo.

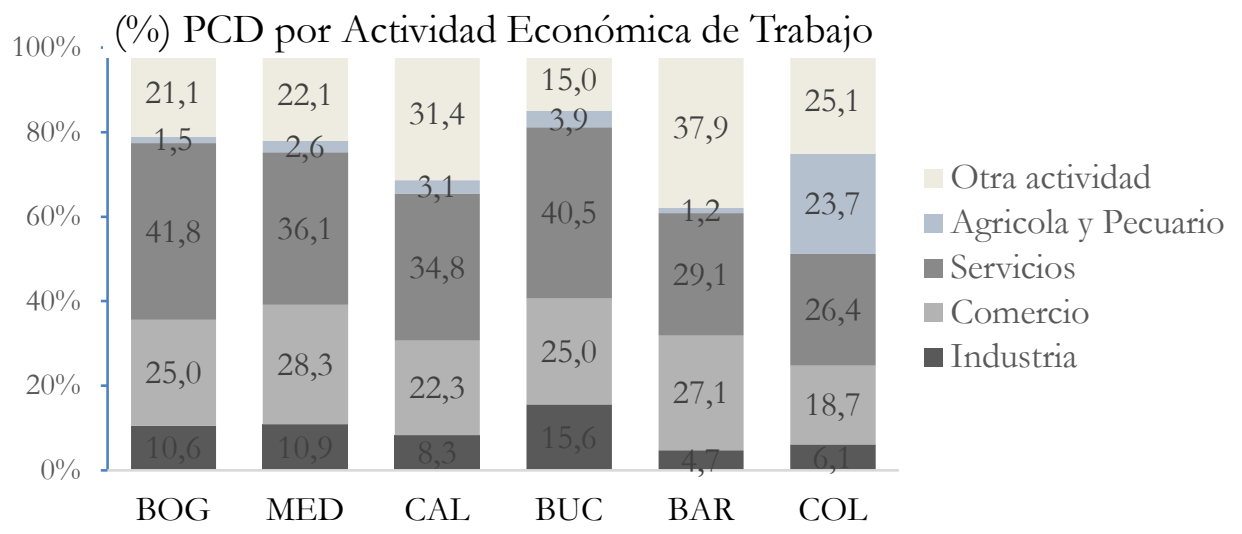

Fuente: Elaboración propia con base en RLCPD (Ministerio de Salud y Protección Social, 2018b).

Por su parte, los niveles de ingreso de la PCD ocupada presentan resultados alarmantes: a nivel de país el 10,6\% de los trabajadores afirmaron no recibir ningún ingreso monetario mensual, mientras que el $71,7 \%$ afirmó percibir salarios de menos de \$500.000 pesos COP (170 USD) que no logran ni siquiera alcanzar el umbral del salario mínimo (SMMLV) fijado en el 2018 en $\$ 781.242$ pesos COP.

A nivel local, Cali presenta una situación más crítica que el país, dado que un 10,9\% de su PCD ocupada no perciben ingresos y el 73,6\% perciben ingresos inferiores a $\$ 500.000$ pesos. Lo que, en conjunto, indica que el $84,5 \%$ de la PCD ocupada de Cali presentan graves problemas para acceder, con su salario laboral, a una cesta básica de bienes y servicios que satisfaga sus necesidades básicas. Así mismo, la ciudad de Bucaramanga presenta hallazgos alarmantes al tener el mayor repunte del grupo en cuanto a PCD ocupada sin ingresos mensuales.

En cuanto a los rangos salariales más altos, destaca Bogotá al situar un 6,8\% de su PCD local en puestos de trabajo con salarios superiores a $\$ 1.500 .000$ pesos COP que equivalen a más de 2 SMMLV del año 2018 (Tabla 4), mientras que Medellín ha reducido a un 3,8\% la PCD ocupada sin ingresos, ampliado además a un nivel de $30,4 \%$ los puestos de trabajo con salarios entre los $\$ 500.000$ y $\$ 1.000 .000$ de pesos COP, que equivalen según el tipo de cambio medio para el 2018 a un rango entre los 170 y 340 USD; por lo que a pesar de presentar críticas tasas de desempleo y ocupación (Tabla 1), su empleo creado está logrando garantizar ingresos que satisfacen adquisitivamente las necesidades básicas de la PCD. 
Tabla 4. Nivel de ingresos (COP).

\begin{tabular}{|c|c|c|c|c|c|c|c|c|c|c|c|c|}
\hline & \multicolumn{12}{|c|}{ PCD Ocupada según ingreso mensual promedio } \\
\hline & BOG & $\%$ & MED & $\%$ & CAL & $\%$ & BUC & $\%$ & BAR & $\%$ & COL & $\%$ \\
\hline Sin Ingreso & 1.545 & 4,1 & 188 & 3,8 & 923 & 10,9 & 436 & 12,7 & 126 & 7,2 & 17.819 & 10,6 \\
\hline Menos de $\$ 500.000$ & 26.334 & 69,5 & 3.046 & 61,1 & 6.220 & 73,6 & 2.317 & 67,4 & 1.158 & 65,9 & 120.214 & 71,7 \\
\hline$\$ 500.001$ a 1.000 .000 & 5.526 & 14,6 & 1.517 & 30,4 & 772 & 9,1 & 510 & 14,8 & 289 & 16,5 & 16.567 & 9,9 \\
\hline$\$ 1.000 .001$ a 1.500 .000 & 1.232 & 3,2 & 113 & 2,3 & 124 & 1,5 & 34 & 1,0 & 28 & 1,6 & 2.830 & 1,7 \\
\hline$\$ 1.500 .001$ a 2.000 .000 & 843 & 2,2 & 36 & 0,7 & 74 & 0,9 & 18 & 0,5 & 7 & 0,4 & 1.470 & 0,9 \\
\hline$\$ 2.000 .001$ a 2.500 .000 & 464 & 1,2 & 19 & 0,4 & 40 & 0,5 & 5 & 0,1 & 7 & 0,4 & 724 & 0,4 \\
\hline Más de $\$ 2.500 .001$ & 1.253 & 3,3 & 28 & 0,6 & 114 & 1,3 & 19 & 0,6 & 7 & 0,4 & 1.723 & 1,0 \\
\hline No reportado & 719 & 1,9 & 36 & 0,7 & 181 & 2,1 & 101 & 2,9 & 134 & 7,6 & 6.382 & 3,8 \\
\hline Total & 37.916 & & 4.983 & & 8.448 & & 3.440 & & 1.756 & & 167.729 & \\
\hline
\end{tabular}

Fuente: Elaboración y estimaciones propias con base en RLCPD (Ministerio de Salud y Protección Social, 2018a).

En la capacidad del mercado laboral para responder a las necesidades laborales de cada grupo de discapacidad se encontró que las cinco categorías de discapacidad con mayor representatividad en Colombia (Movilidad (19,8\%), Mental-Cognitivo (10,5\%), Múltiple (8,4\%), Visual (5,4\%), Auditiva $(3,6 \%))$, en razón al tamaño de su población, coinciden con los grupos que en términos proporcionales han logrado una mayor inserción. Tal situación se repite exactamente para Medellín y Barranquilla, mientras que Bucaramanga, Bogotá y Cali sólo difieren al incluir entre los cinco grupos categóricos la PCD Sistémica en lugar de la PCD Mental-Cognitiva. Así mismo, también se cumple que los grupos con menor representatividad en tamaño coinciden con los grupos con menor ocupación laboral, a pesar de que algunos de estos grupos minoritarios presentan barreras muy altas de exclusión por el tipo de discapacidad como el caso de PCD de voz y habla (Tabla 5).

Tabla 5. Ocupación y patología.

\begin{tabular}{|c|c|c|c|c|c|c|}
\hline \multirow[b]{2}{*}{ Categoría Discapacidad } & \multicolumn{6}{|c|}{$(\%)$ de PCD trabajando en los últimos 6 meses } \\
\hline & BOG & MED & CAL & BUC & BAR & COL \\
\hline Auditiva & 2,1 & 5,7 & 2,4 & 1,9 & 9,7 & 4,4 \\
\hline Enanismo & 0,0 & 0,1 & 0,0 & N/A & 0,1 & 0,1 \\
\hline Mental cognitivo & 1,1 & 5,6 & 0,7 & 0,9 & 3,6 & 2,6 \\
\hline Mental psicosocial & 1,3 & 4,8 & 0,8 & 0,4 & 1,8 & 1,3 \\
\hline Movilidad & 12,3 & 28,2 & 11,1 & 8,2 & 44,1 & 19,6 \\
\hline Múltiple & 3,6 & 9,0 & 1,3 & 1,5 & 5,2 & 3,3 \\
\hline No la sabe nombrar & 0,4 & 0,9 & 0,6 & 0,8 & 2,2 & 1,7 \\
\hline No reportado & 75,6 & 36,9 & 76,6 & 81,4 & 20,4 & 57,5 \\
\hline Piel, uñas y cabello & 0,1 & 0,1 & 0,1 & 0,2 & 0,4 & 0,2 \\
\hline Sensorial gusto-olfato-tacto & 0,1 & 0,1 & 0,1 & 0,2 & 0,3 & 0,2 \\
\hline Sistémica & 1,8 & 2,0 & 1,6 & 1,9 & 2,3 & 1,9 \\
\hline Visual & 1,7 & 6,0 & 4,2 & 2,3 & 9,1 & 6,1 \\
\hline Voz y habla & 0,1 & 0,5 & 0,5 & 0,4 & 1,0 & 1,2 \\
\hline Total Muestra & 37.916 & 4.983 & 8.448 & 3.440 & 1.756 & 167.729 \\
\hline Coef. de Variación Estandarizado* & $\mathbf{0 , 6 8}$ & 0,49 & $\mathbf{0 , 6 0}$ & $\mathbf{0 , 4 4}$ & $\mathbf{0 , 8 4}$ & $\mathbf{0 , 5 2}$ \\
\hline
\end{tabular}

*Para el cálculo se eliminaron los datos del grupo "No reportado" y se procedió a estandarizar los resultados para reportarlos en los mismos rangos de evaluación.

Fuente: Elaboración y estimaciones propias con base en RLCPD (Ministerio de Salud y Protección Social, 2018a). 
En efecto, tal situación es alarmante ya que, si el grado de priorización para responder a las diferentes necesidades de empleo de cada tipo de discapacidad obedece únicamente a criterios de representatividad demográfica, se podría estar incidiendo gravemente en el incremento de disparidades entre la misma población.

Para Colombia la mayor ocupación la presenta la categoría de PCD con movilidad con un 19,6\% del total de los trabajadores, seguido de la PCD visual, aunque con una cifra notablemente inferior del $6,1 \%$. A nivel local, la PCD de movilidad también presenta las mayores tasas de participación con especial relevancia para Barranquilla, que además ha logrado incluir notablemente a la PCD auditiva con un nivel de $9,7 \%$, siendo éste su segundo grupo con mayor inserción.

Así mismo, Barranquilla presenta el mayor coeficiente de variación, lo que implica que los niveles de ocupación en esta ciudad son más heterogéneos entre los grupos de discapacidad, por lo que la PCD de Barranquilla debe enfrentarse a una mayor desigualdad para emplearse debido al tipo de discapacidad que presente.

A nivel de capacitación para el trabajo, se encuentra que 24.725 personas de las cinco ciudades de análisis han recibido capacitación; no obstante, en contra posición se encuentran un grupo diez veces más grande, equivalente a 252.987 personas, que no han sido atendidas pese a manifestar requerimientos de tales servicios. Además, las ciudades brindaron mayor cobertura de capacitación para el grupo de PCD de movilidad (3.224), de carácter mental cognitivo (1.767) y con características múltiple (1.489), mientras que, contrastando los niveles de capacitación efectiva y requerida, se encuentra una especial falta de atención de las ciudades en capacitación para la PCD mental psicosocial, sistémica y de voz y habla (Tabla 6).

Tabla 6. Capacitación para el trabajo.

\begin{tabular}{|c|c|c|c|c|c|c|c|c|c|c|}
\hline \multirow{3}{*}{\begin{tabular}{|l} 
\\
Categoría \\
\end{tabular}} & \multicolumn{10}{|c|}{$\begin{array}{c}\text { Capacitación para el trabajo } \\
\text { Población con Requerimiento }(*) \text { - población atendida (PA) }\end{array}$} \\
\hline & \multicolumn{2}{|c|}{ BOG } & \multicolumn{2}{|c|}{ MED } & \multicolumn{2}{|c|}{ CAL } & \multicolumn{2}{|c|}{ BUC } & \multicolumn{2}{|c|}{ BAR } \\
\hline & $*$ & $P A$ & $*$ & $P A$ & $*$ & $P A$ & $*$ & $P A$ & $*$ & $P A$ \\
\hline Auditiva & 2.193 & 327 & 1.324 & 332 & 946 & 174 & 730 & 43 & 773 & 159 \\
\hline Enanismo & 18 & 4 & 37 & 12 & 10 & 2 & 5 & 1 & 15 & $\mathrm{~N} / \mathrm{A}$ \\
\hline Mental cognitivo & 5.230 & 507 & 5.491 & 801 & 2.033 & 144 & 632 & 129 & 1.865 & 186 \\
\hline Mental psicosocial & 2.577 & 212 & 2.519 & 341 & 677 & 48 & 858 & 11 & 620 & 49 \\
\hline Movilidad & 12.837 & 1.218 & 8.151 & 1.087 & 5.875 & 465 & 2.900 & 97 & 3.953 & 357 \\
\hline Múltiple & 9.097 & 752 & 4.409 & 479 & 1.613 & 113 & 629 & 58 & 1.564 & 87 \\
\hline No la sabe nombrar & 450 & 36 & 166 & 24 & 399 & 20 & 80 & 14 & 289 & 29 \\
\hline No reportado & 108.771 & 10.638 & 11.188 & 940 & 29.927 & 2.590 & 5.829 & 742 & 2.224 & 134 \\
\hline Piel, uñas y cabello & 43 & 16 & 40 & 3 & 48 & 4 & 23 & 3 & 35 & 5 \\
\hline $\begin{array}{l}\text { Sensorial (gusto-olfato- } \\
\text { tacto) }\end{array}$ & 63 & 6 & 22 & 2 & 39 & 2 & 16 & & 20 & $\mathrm{~N} / \mathrm{A}$ \\
\hline Sistémica & 2.495 & 160 & 1.014 & 111 & 1.017 & 68 & 637 & 81 & 465 & 28 \\
\hline Visual & 2.338 & 283 & 1.501 & 232 & 2.006 & 146 & 505 & 33 & 912 & 102 \\
\hline Voz y habla & 153 & 20 & 167 & 17 & 200 & 26 & 118 & 4 & 206 & 11 \\
\hline Total & 146.265 & 14.179 & 36.029 & 4.381 & 44.790 & 3.802 & 12.962 & 1.216 & 12.941 & 1.147 \\
\hline PR/PCD Muestral" (\%) & \multicolumn{2}{|c|}{53,9} & \multicolumn{2}{|c|}{56,1} & \multicolumn{2}{|c|}{70,6} & \multicolumn{2}{|c|}{$\mathbf{5 7 , 9}$} & \multicolumn{2}{|c|}{65,1} \\
\hline PA/PCD Muestral ${ }^{* *}(\%)$ & \multicolumn{2}{|c|}{5,2} & \multicolumn{2}{|c|}{6,8} & \multicolumn{2}{|c|}{5,9} & \multicolumn{2}{|c|}{5,4} & \multicolumn{2}{|c|}{5,7} \\
\hline
\end{tabular}

*Número total de personas que respondieron a la pregunta ¿Necesita o no capacitación para mejorar o cambiar su actividad laboral productiva? ${ }^{* *}$ Número total de personas que respondieron a la pregunta ¿Ha recibido o no capacitación para el trabajo?

Fuente: Elaboración propia con base en RLCPD - SISPRO (Ministerio de Salud y Protección Social, 2018a). 
También se obtiene que Cali es la ciudad con mayor porcentaje de PCD con requerimientos de capacitación para el trabajo que no ha sido atendida, alcanzando una tasa de $70,6 \%$ equivalentes a 44.790 personas, mientras que Medellín presenta la mayor cobertura en capacitación para el trabajo con una población atendida de 6,8\% y en la última posición se ubica Bogotá con una atención del 5,2\%.

No obstante, tales hallazgos denotan coberturas muy bajas que dejan en evidencia graves problemáticas del escenario local para incrementar las competencias laborales de forma que se incida en la empleabilidad y se reduzcan las disparidades entre la oferta y demanda de trabajo, tales como la probabilidad de encontrar trabajo y la probabilidad de que efectivamente se ocupe una vacante creada. No obstante, a continuación, se construye una función de emparejamiento laboral de la PDC para determinar la magnitud de tales incidencias.

\section{El emparejamiento en el mercado laboral de la PCD.}

La función de emparejamiento 'matching function' nos muestra la relación existente entre las vacantes y los desempleados. Ésta es una función agregada que depende de la cantidad de firmas que contratan trabajadores en un momento dado, de los trabajadores que buscan empleo y, en algunas ocasiones, de otros factores que se incorporan dependiendo del modelo utilizado.

A partir de la teoría de la búsqueda de Stigler (1962), se desarrollaron una serie de modelos de búsqueda secuenciales y más dinámicos (Mortensen, 1986). Estos modelos comparan la situación del individuo en estado de desempleo con la situación de tener un empleo teniendo en cuenta el salario de reserva del individuo en el momento de evaluar esa posibilidad de emplearse. Sin embargo, la incorporación de fricciones en el mercado y el papel del empleador (demanda laboral) en la determinación de los salarios y del desempleo, llevó a Mortensen y Pissarides (1999) a mostrar "que existen dos tendencias en los modelos de búsqueda, pero pueden consolidarse en una sola cuando pasa el tiempo" (Mora, Castillo, Castro \& Raffo, 2018 p. 529). Por otro lado, estas fricciones, para el caso colombiano, son explicadas principalmente por la dispersión del desempleo regional y por la deficiencia en la gestión de la intermediación (Riveros, 2017)”.

Es así, como los agentes se encuentran en el mercado laboral a través de una función de emparejamiento con la presencia de fricciones, instituciones e inversiones óptimas por parte de los agentes (Mortensen \& Pissarides, 1999). Ahora bien, como lo plantea Pissarides (2000) es imposible la presencia de un emparejamiento total entre oferta y demanda laboral y, por esta razón, existirán en algunas ocasiones más vacantes que colocados para algún tipo de ocupación. Y se puede aumentar la eficiencia en el proceso de emparejamiento a través de mejoras en los procesos de búsqueda y registro de las vacantes.

Cabe observar que otros autores han demostrado que las interacciones espaciales afectan el emparejamiento de los mercados locales de trabajo, hallando significativos los efectos de proximidad regional (Antczak, Gałecka \& Pater, 2018; Fedorets, Lottmann \& Stops, 2019), y que tanto el desajuste de habilidades como el desajuste espacial, que dependen de las características de la economía local, los empleadores, los solicitantes de empleo y los trabajos que se están considerando, son importantes para explicar el éxito de la búsqueda de empleo (McQuaid, 2006).

A continuación, supongamos que $H$ sea el número de contrataciones de $\mathrm{PCD}$, que depende del número de desempleados con discapacidad $(U)$ y del número de vacantes para personas con discapacidad $(V)$. Entonces la función de emparejamiento se puede plantear de la siguiente forma:

$$
H=M(U, V)
$$


Dado que $\mathrm{H}$ es creciente en $\mathrm{U}$ y en $\mathrm{V}$, entonces $\frac{\partial M(U, V)}{\partial V}$ serán positivas. También supondremos que $\mathrm{H}$ es cóncava en ambas variables y homogénea de grado 1 .

A continuación sea $L$ la cantidad de personas con discapacidad, de tal forma que $M / L=m$ represente la tasa de emparejamiento con respecto a $L, U / L=u$ sea la tasa de desempleo y $V / L=v$ sea la tasa de vacantes. Entonces el modelo econométrico a estimar será:

$$
\ln (m)_{j}=\alpha_{0}+\alpha_{u} \ln (u)_{j}+\alpha_{v} \ln (v)_{j}+\varepsilon_{j}
$$

donde $\alpha$ y $\beta$ son los parámetros de la función de emparejamiento siguiendo una Cobb-Douglas y $\mathrm{j}$ denota un departamento en particular.

En Colombia, Mora y Santacruz (2007) y Castillo, Castro, Raffo y Mora (2018), entre otros, han realizado estas estimaciones utilizando datos de panel.

Castro (2017) estimó la función de emparejamiento para el mercado laboral colombiano a partir de datos del Servicio Público de Empleo (SPE) encontrando que existe una externalidad positiva más alta para los desempleados que para las vacantes; de tal manera sus hallazgos arrojaron que la probabilidad promedio para que un desempleado colombiano encuentre trabajo es del $86 \%$ y la probabilidad promedio de que se ocupe una vacante es del 14\%. Este resultado puede ser diferente para las personas con discapacidad y afectar en mayor medida la probabilidad de que un desempleado encuentre trabajo, así como la probabilidad de que una vacante sea ocupada. En esta vía, Agovino y Rapposelli (2017) encontraron un efecto de congestión entre las personas desempleadas con discapacidad, debido al exceso de personas discapacitadas desempleadas en comparación con las vacantes disponibles.

Coles y Smith (1996) estiman la función de matching utilizando datos de corte transversal de desempleo, vacantes y colocación de empleo a nivel de ciudades para el Reino Unido y encuentran que las estimaciones son similares a las realizadas por Blanchard y Diamond (1994) a nivel agregado encontrando también rendimientos constantes a escala. Es por ello, que la estimación que aquí realizaremos de corte transversal es igual de válida a la de datos de panel.

A partir de los resultados de la ecuación (2) es posible encontrar los siguientes tipos de rendimientos:

Tabla 7. Rendimientos a escala de la función de emparejamiento.

\begin{tabular}{|c|c|c|}
\hline Parámetros & Tipo de rendimiento & Conclusión \\
\hline$\alpha_{u}+\alpha_{v}>1$ & Crecientes & $\begin{array}{c}\text { Aumentar el nivel de búsqueda genera que el proceso de } \\
\text { emparejamiento sea más eficiente. }\end{array}$ \\
\hline$\alpha_{u}+\alpha_{v}<1$ & Decrecientes & $\begin{array}{c}\text { Aumentar el nivel de búsqueda genera que el proceso de } \\
\text { emparejamiento sea menos eficiente. }\end{array}$ \\
\hline$\alpha_{u}+\alpha_{v}=1$ & Constantes & $\begin{array}{c}\text { Todos los buscadores tienen igual probabilidad de ser } \\
\text { contratados. }\end{array}$ \\
\end{tabular}

Fuente: Adaptado de Castillo, Castro, Raffo \& Mora (2018) y Mora \& Santacruz (2007).

El análisis del tipo de rendimientos a escala es fundamental ya que determina el tipo de impacto que pueden tener las políticas laborales encaminadas a minimizar las fricciones en el mercado laboral, a incentivar ampliaciones en la escala, o a mejorar la velocidad de rotación de los mercados. Bajo la existencia de rendimientos crecientes o decrecientes las políticas inciden sobre el nivel de eficiencia de los procesos de búsqueda de empleo, mientras que cambios en la escala o en la intensidad de búsqueda 
por parte de oferentes y demandantes influyen sobre la tasa de emparejamiento. De esta forma, cuando existen rendimientos crecientes, el impacto de las políticas permite incrementar el nivel de eficiencia en los procesos de búsqueda.

A partir de las estadísticas por departamento del Servicio Público de Empleo de Colombia sobre la PCD que está buscando empleo, la PCD contratada y el número de vacantes que ofrecen las empresas para la población con discapacidad en el año 2018, se procedió a realizar la siguiente regresión de emparejamiento (cabe observar que no se pudieron incluir todos los departamentos ya que para el 2018 no existe información de vacantes, inscritos y colocados de todos estos, por lo que solo se incluyeron 27 departamentos):

Tabla 8. Resultados de la estimación por departamento de la PCD.

\begin{tabular}{|cc|}
\hline Desempleados/L & $0,394896^{*}$ \\
$(0,157881)$ & $1,118760^{* * *}$ \\
Vacantes/L & \\
$(0,159554)$ & $1,720775^{*}$ \\
Constante & \\
$(0,864609)$ & 0,574 \\
\hline R-Cuadrado & 118,649 \\
chi2 & 27 \\
\hline $\mathrm{N}$ & \\
\hline
\end{tabular}

Fuente: Cálculos propios a partir de datos del SPE. ${ }^{*} \mathrm{p}<0,05,{ }^{* *} \mathrm{p}<0,01,{ }^{* * *} \mathrm{p}<0,001$.

Los resultados (Tabla 8) muestran que las variables son estadísticamente significativas al 95\% en el caso del desempleo y la constante, y al $99 \%$ en el caso de las vacantes. Por otro lado, se contrastó la hipótesis de que existieran rendimientos constantes a escala $\alpha+\beta=1$ que se rechaza ya que la chi cuadrado con un grado de libertad fue de 12,99 y un p-valor de 0,0003 . También se rechazó la hipótesis de que conjuntamente la suma sea igual a cero $(\operatorname{chi} 2(1)=12,83$ con Prob $>\operatorname{chi} 2=0,0000)$. Se utilizó Boostraping con el fin de obtener errores estándar robustos ante atípicos.

De esta forma, los resultados muestran la existencia de rendimientos crecientes en la función de emparejamiento $(\alpha+\beta=1,5136561)$. Los resultados también muestran como, en promedio, la probabilidad de que un trabajador con discapacidad encuentre trabajo es de un $26,1 \%\left(\frac{\alpha_{u}}{\alpha_{u}+\alpha_{v}}\right)$ y la probabilidad promedio de que se ocupe una vacante para un trabajador con discapacidad es del $73,9 \%$ $\left(\frac{\alpha_{\mathrm{v}}}{\alpha_{\mathrm{u}}+\alpha_{\mathrm{v}}}\right)$.

En resumen, estas asimetrías con respecto a las probabilidades de encontrar trabajo y de ocupar vacantes determinan la necesidad de establecer políticas activas de empleo con enfoque local que mejoren los procesos y las competencias blandas de búsqueda de empleo para la PCD en Colombia.

No obstante, en los casos de estudio ya se presentan algunas iniciativas de empleo que consideran el contexto local como una variable clave para la efectividad de las políticas. Estas iniciativas han sido instrumentalizadas principalmente a través de programas que presentan asociatividad de actores públicos y privados, y destacan actualmente como buenas prácticas para reducir las asimetrías entre la demanda y la oferta de trabajo. Algunas de las principales medidas se revisan a continuación. 


\section{Iniciativas locales de empleo para PCD con buenas prácticas para reducir las asimetrías del mercado laboral.}

Si bien las políticas de empleabilidad en Colombia siguen directrices de ámbito nacional, es importante destacar que las ciudades están adaptando medidas descentralizadas con enfoques de desarrollo local, que pese a su incipiente avances, presentan una oportunidad para configurar estructuras ascendentes y territoriales que permitan una gestión capaz de actuar en concordancia con los elementos, recursos, actores y oportunidades confluentes en cada mercado local de trabajo, logrando así gestionar las asimetrías entre la oferta y demanda de trabajo y con ello incrementar la empleabilidad de la PCD. Entre los casos locales más exitosos se encuentran los siguientes.

\subsection{El Servicio Público de Empleo (SPE).}

Creado por la Ley 1636 de 2013 como mecanismo de soporte al cesante en Colombia y herramienta para la búsqueda de empleo, el SPE opera bajo la Unidad del Servicio Público de Empleo (UAESPE, encargada de gestionar y dirigir los recursos, servicios e instrumentos públicos de gestión y colocación del empleo del SPE y de su red de prestadores, así como de administrar la información de la oferta y la demanda del mercado laboral del Sistema de Información del Servicio de Empleo), y tiene como objetivo mejorar la empleabilidad gestionando de manera sistemática las asimetrías entre la oferta y la demanda de trabajo a través de una ruta de actuación en 4 fases (registro, orientación, intermediación y gestión empresarial) que generan importantes impactos a nivel territorial, empresarial y poblacional. Concretamente, esta iniciativa presenta una dimensión estratégica interorganizacional que favorece el fomento del empleo local para PCD, esto al presentar una estructura operativa centrada en la articulación de actores a escala local (que logra asociatividad entre prestadores de carácter público constituidos como centros de empleo y prestadores privados de apoyo como agencias privadas lucrativas, no lucrativas y bolsas universitarias de empleo) y una intervención con enfoque diferencial para la PCD (que logra adaptar estrategias tales como el seguimiento público de oferentes, la caracterización periódica de la oferta de trabajo inclusivo, la capacitación a la red de prestadores de servicios en procesos de atención, adaptación y promoción laboral para PCD, así como el otorgamiento de herramientas suficientes para asesorar al sector empresarial en los ajustes y requerimientos específicos que deben aplicarse para poder vincular eficazmente a la PCD. Implementación de teletrabajo, uso de tecnologías de la información y las comunicaciones, capacitación y uso del servicio de interpretación en línea Centro de Relevo, adecuación del espacio de trabajo en requerimientos de movilidad, accesibilidad, iluminación y señalización, flexibilidad en los horarios para casos específicos, entre otros (Corona et al., 2017)).

En cuanto a los avances de atención a PCD dentro del SPE (análisis con base en la información aportada al estudio por la UAESPE del Servicio Público de Empleo), se encontró para las cinco ciudades de estudio un total de 1.428 personas registradas en el programa, pero con una concentración del 57,8\% de los casos para la ciudad de Bogotá y de solo el 5,0\% para la ciudad de Bucaramanga, lo que sigue reflejando las altas brechas de atención a la discapacidad entre las diferentes ciudades. Por su parte, los resultados del SPE en orientación (1.342 beneficiarios para el grupo de ciudades) y colocación (15.838 beneficiarios para el grupo de ciudades) demuestran una notable efectividad del SPE para reducir las asimetrías entre la oferta y demanda de trabajo para PCD, pero continúa con un alcance altamente concentrado en la atención de la PCD de la ciudad de Bogotá, por lo que el programa debe implementar medidas urgentes para descentralizar la atención de sus servicios para PCD y lograr equiparar los resultados entre las distintas ciudades.

Así mismo, Bogotá concentra el mayor número de prestadores del SPE al reunir un total de 35 operadores locales, seguido de Medellín 18, Barranquilla 10, Bucaramanga 9 y, por último, Cali presenta sólo 8 operadores a pesar de ser la tercera ciudad del país con mayor tamaño de PCD (Tabla 9). 
Tabla 9. Intervención local: Servicio Público de Empleo (SPE).

\begin{tabular}{|c|c|c|}
\hline \multirow{2}{*}{ Operativa } & Centros de empleo & BOG \\
\cline { 2 - 3 } & Puntos de Servicio & 19 \\
& Bolsas de Universidades & 5 \\
\hline \multirow{2}{*}{ Territorial } & Alianza con entidades, consecución de operadores locales, análisis del mercado local \\
& de trabajo, gestión con alcaldías y gobernación. \\
\hline Empresarial & Gestión, Sensibilización, Diagnóstico, Remisión de Personas. \\
\hline Poblacional & Orientación, formación, intermediación, entrega de recursos. \\
\hline
\end{tabular}

Fuente: Elaboración propia con base en Ministerio de Trabajo (2018) y CORONA, ANDI \& ACDI VOCA

(2016).

\subsection{Securitas, Programa de Vigilancia Inclusiva (PVI).}

Este programa hace parte de la estrategia principal de Responsabilidad Social Corporativa de Securitas Colombia S.A. y opera desde el año 2007 en la ciudad de Bogotá con planes de expansión para las ciudades de Cali, Barranquilla y Medellín; el objetivo principal del PVI es la vinculación y permanencia de la PCD física en el mercado laboral de servicios de vigilancia ofrecidos a distintas empresas, de forma que se generen impactos en la calidad de vida de la PCD y se fomente el empleo inclusivo (Securitas Colombia, 2018).

El PVI se destaca por la capacitación y seguimiento dirigido a la PCD a emplear y a los clientes empresariales donde las personas con discapacidad desarrollarán su actividad laboral, generando por tanto un incremento de la formación para el trabajo de la PCD mientras reduce además las barreras de acceso dentro de otras empresas.

Entre los resultados, la PCD física contratada ha pasado de 11 personas en el 2007 a 86 personas en el 2016, año para el que 70 de los empleados fueron mujeres (Tabla 10) evidenciando, por tanto, un crecimiento en la contratación de la PCD acompañado de un notable enfoque de género. Así mismo, se encontró que el programa ha logrado aplicarse en siete organizaciones de Bogotá (Securitas Colombia, 2016), mientras que, las entrevistas aplicadas a directivos y beneficiarios del programa dejaron en evidencia que el PVI ha permitido un importante cambio de paradigmas entre demandantes y ofertantes del sector, que ha motivado la participación y el reconocimiento de la PCD y con ello el incremento de la demanda de su empleo.

Tabla 10. Intervención local: Securitas, Programa de Vigilancia Inclusiva (PVI).

\begin{tabular}{|c|c|c|c|c|c|c|}
\hline \multirow{3}{*}{ Operativa } & & \multicolumn{5}{|c|}{ BOG } \\
\hline & & 2012 & 2013 & 2014 & 2015 & 2016 \\
\hline & Empleos creados & 53 & 62 & 66 & 71 & 86 \\
\hline Territorial & \multicolumn{6}{|c|}{$\begin{array}{l}\text { Especialización de la PCD física en el sector de servicios, alianza con entidades de apoyo, } \\
\text { consecución de clientes locales. }\end{array}$} \\
\hline Empresarial & \multicolumn{6}{|c|}{$\begin{array}{c}\text { Sensibilización, capacitación, diagnóstico, implementación de ajustes, acompañamiento a } \\
\text { la empresa. }\end{array}$} \\
\hline Poblacional & \multicolumn{6}{|c|}{ Vinculación laboral directa, formación y seguimiento. } \\
\hline
\end{tabular}

Fuente: Elaboración propia con base en Securitas Colombia (2016) y CORONA, ANDI \& ACDI VOCA (2016). 


\subsection{Programa Pacto de Productividad (PP).}

Éste es un programa empresarial de promoción laboral para la PCD fundado en el año 2009 a través de la alianza estratégica de una red de instituciones públicas y privadas (Fundación Corona, Fundación Saldarriaga Concha, Banco Interamericano de Desarrollo, Agencia Presidencial de Cooperación Internacional de Colombia (APC), Ministerio del Trabajo, Servicio Nacional de Aprendizaje (SENA), Cafam - Bogotá, Comfenalco Antioquia - Medellín, Comfandi - Cali, Comfamiliar Risaralda - Pereira), con apoyo financiero del Fondo Multilateral de Inversiones del Banco Interamericano de Desarrollo (FOMIN-BID) y la responsabilidad como principal ejecutor de la Fundación Corona. El costo del programa en la Fase I (2009-2014) fue de 3,2 millones de USD, entre los que 54\% fueron contribuidos por FOMIN-BID (FOMIN-BID, 2015).

El PP ha sido dividido en dos fases de intervención, la Fase 1 (2009-2014) tuvo operatividad local en las ciudades de Bogotá, Cali, Medellín y Pereira, teniendo como objetivo diseñar un modelo concertado de inclusión laboral para PCD de Colombia en capacidad de mejorar las oportunidades, la cualificación y la promoción de empleo de la población objetivo a través de la actuación articulada de actores con incidencia directa o indirecta sobre los mercados locales de trabajo, mientras que la Fase II (2014 - actualidad) tiene como objetivo la transferencia e implementación del modelo de inclusión laboral de manera efectiva, productiva y con calidad en el ámbito nacional.

Entre las características a destacar del programa se encuentra el alcance de mercado resultado de la implicación y vinculación de distintos actores locales públicos y privados; la participación de mesas locales categóricas de discapacidad que permiten la participación activa de los distintos grupos de discapacidad y definir intervenciones consensuadas con la población objetivo para lograr una mejor adaptabilidad de los requerimientos que necesita cada tipo de discapacidad; el desarrollo de un modelo de inclusión laboral con extrapolación nacional en el largo plazo y aplicabilidad adaptada al contexto local y a los requerimientos de las distintas categorías de discapacidad y, finalmente, un pacto interorganizacional con estructura de clúster social que establece un compromiso de movilización interorganizacional y cooperativo para la aplicación del modelo de inclusión y asegura el sostenimiento y la maximización al largo plazo de los procesos de empleabilidad generados por el programa.

Los impactos del programa PP en su Fase I (análisis con base en los aportes de la Dirección del Programa Pacto de Productividad y en los datos recopilados en Pacto de Productividad (2015) y FOMIM-BID (2015)) muestran una notable eficacia en su alcance. Entre los principales logros se encuentran:

- A nivel de costo-efectividad, en el año 2015 los salarios de la PCD insertada laboralmente por empresas vinculadas al programa PP fueron equivalentes a $\$ 5.219,4$ millones de COP (2,5 millones de USD aproximadamente), cifra equivalente al $75 \%$ del costo total del programa que se aproxima en 3,2 millones de USD.

- A nivel empresarial, PP generó directamente más de 1.000 servicios de intermediación laboral a 192 empresas cuya inversión es equivalente a los \$385 millones de COP, así mismo, el conjunto de empresas presentó un ahorro aproximado de $\$ 2.825$ millones de COP al acceder a beneficios de ley como deducciones de renta, ahorros en cuotas de aprendiz e incluso aprendices sin costos, después de haber sido capacitadas por el programa.

- A nivel institucional, el PP ha generado servicios por más de \$508 millones de COP al SENA y un ahorro aproximado de más de $\$ 2.000$ millones de COP, además de asesoría directa en el año 2013 a 11 Centros Públicos de Empleo, así como una alta transferencia de conocimiento a la Unidad del Servicio Público de Empleo (UAESPE).

- A nivel de políticas públicas, el PP apoyó la redacción e implementación de la Política Institucional de discapacidad del SENA 2014-2018, asesoró la formulación de la Ley 1618 de 2013 reglamentada 
en el 2014 y del CONPES 166 del sistema nacional de discapacidad, y aportó al Manual de valoración ocupacional de personas con discapacidad del Ministerio de Salud y Protección Social.

- A nivel local, la efectividad del programa medida por los salarios generado para PCD se distribuyen en $23,28 \%$ para Cali, $20,45 \%$ para Bogotá y $18,31 \%$ para Medellín, mientras que el número de PCD beneficiaria es de 494 personas para la ciudad de Medellín, 483 para Cali y 309 personas para Bogotá.

Así mismo, entre las ciudades se evidenció como efecto de la Fase I del programa, una especialización en atención a los grupos categóricos de discapacidad que presentaban mayores problemas en el contexto local, de tal manera el 46,3\% de los beneficiarios en Medellín fueron PCD cognitiva, mientas que Cali y Bogotá atendieron en $44,3 \%$ y $69,2 \%$ a PCD auditivita.

Además, analizando en conjunto los resultados (Tabla 11), Cali presentó el impacto local más importante del programa PP al repuntar en personas beneficiarias, beneficiarios de formación, empresas con personas contratadas, vacantes disponibles generadas, además de ser la ciudad con mayor participación sobre los salarios generados por el programa. No obstante, el programa PP tiene como reto disminuir la alta tasa de deserción de los programas formativos que alcanza la ciudad de Cali.

Tabla 11. Intervención local: Programa Pacto de Productividad (PP).

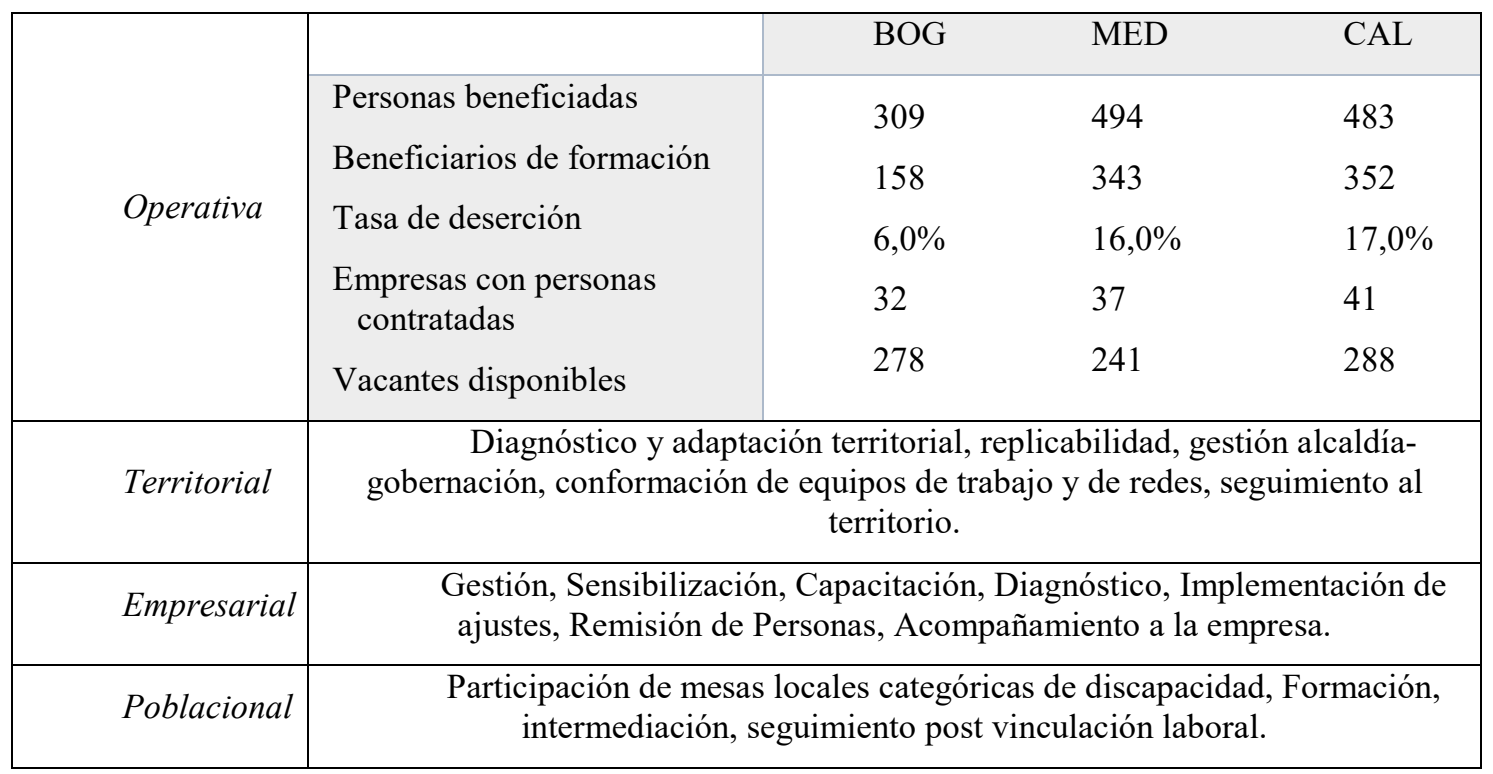

Fuente: Elaboración propia con base en Pacto de Productividad (2015), FOMIM-BID (2015) y CORONA, ANDI Y ACDI VOCA (2016).

La Fase II (2015-acualidad) presenta impactos complementarios en las cuatro ciudades iniciales de intervención del programa, así como una importante transferencia de conocimiento del modelo inclusivo para que éste sea adaptado como modelo de gestión y atención a población vulnerable dentro del Servicio Público de Empleo (4.1), logrando una extensión a otras ciudades donde tiene presencia el programa y, por tanto, consolidando una red de aliados estratégicos en todo el escenario nacional. Según la información aportada por la Dirección del Programa Pacto de Productividad a finales del 2018, los principales avances de la Fase II se relacionan con asesorías y acompañamiento a agencias de empleo del SPE obteniendo los siguientes avances:

- Formación y transferencia de conocimiento del modelo a la red de prestadores del SPE logrando cobertura para 67 municipios, 135 centros de empleo, 36 funcionarios y 687 gestores. 
- Intervención en 2 centros públicos de empleo adicionales a los 11 de la Fase I, así como asesorías a 8 empresas adicionales.

- Publicación de guías de protocolo de atención para gestores y para empresarios.

- Implementación de ajustes y mejoras a los servicios de 4 centros de empleo pilotos del programa para garantizar el acceso a la PCD.

- Actividades para adaptar los procesos, procedimientos y prácticas para la atención e inclusión de la PCD Física, visual, auditiva, visual y auditiva, cognitiva y psicosocial.

- Creación en 2018 del proyecto "Fondo de Oportunidades para el Empleo" dirigido a consolidar la apropiación de conocimiento del modelo de inclusión laboral con enfoque en el cierre de brechas y articular una red de Centros de Empleo Inclusivos.

- Intervenciones específicas en instituciones públicas y privadas de las cinco ciudades de análisis en temas como fortalecimiento de capacidades de gestión empresarial, ajustes razonables del modelo de inclusión y mitigación de barreras.

Finalmente, el programa PP ha iniciado un seguimiento a empresas de la ciudad de Bogotá que han sido acogidas por el programa en años anteriores, actualmente se han visitado 15 empresas seleccionadas, identificando 6 con prácticas activas de contratación, 5 con implementación de políticas de inclusión, 13 con procesos de inclusión laboral desde sus estrategias de Responsabilidad Social Corporativa, notables ajustes en flexibilización de requisitos por parte de gestión humana y uso de canales de interpretación, 12 empresas con necesidad de nuevas capacitaciones, así como 77 de 105 personas con continuidad laboral, lo que indica un sostenimiento de los empleos creados para PCD del $73,3 \%$.

\section{Conclusiones.}

Todas las ciudades presentan políticas públicas locales de discapacidad con distintos grados de intervención en materia de empleabilidad para PCD, Bogotá aprobó su política de discapacidad en el año 2007, mientras que Barranquilla la aprobó en el año 2015 tras un proceso de construcción colectiva. De igual manera se encontraron directrices nacionales y legislación sobre incentivos que buscan la formalización y fomento del empleo inclusivo para la PCD. Entre tales medidas se encontraron la obligatoriedad de cuotas de empleo para instituciones públicas locales que oscilan entre el $0,5 \%$ y $2,0 \%$ como meta para el año 2019; directrices para las distintas entidades en formulación de programas públicos de empleo inclusivo; normativas para el teletrabajo en garantía de los derechos laborales; y un amplio paquete de incentivos para las empresas inclusivas entre los que se encuentran deducciones y descuentos en el impuesto sobre la renta, descuentos complementarios de los aportes parafiscales, preferencia en procesos de contratación pública, reducciones en la cuotas obligatorias de aprendices, descuentos arancelarios sobre la importación de maquinaria y equipo especializado para la PCD, así como preferencias en licitaciones, préstamos y subvenciones del estado.

Respecto a los anteriores aspectos, se encontraron avances a nivel local en las cuotas de empleo para PCD en las instituciones públicas de las ciudades, especialmente en las de Bogotá y Cali que presentaron un $7,4 \%$ y $5,1 \%$ de empleados con discapacidad. No obstante, las cifras disponibles son para el conjunto local de instituciones públicas y, por tanto, tales resultados no implican que a nivel per cápita las instituciones estén cumpliendo con las cuotas exigidas por la ley.

El sector de servicios es el más incluyente en términos de la capacidad para generar empleo dirigido a la PCD. No obstante, el conjunto de ciudades de análisis, a pesar de concentrar el principal 
aparato productivo y económico del país, tiene tasas de trabajadores por cuenta propia más altas que el promedio nacional, aunque bien con una menor tasa de trabajadores familiares sin remuneración.

Sin embargo, las ciudades presentan generalizadamente signos alarmantes. A nivel de desempleo Barranquilla, Cali y Medellín superan de manera crítica a la media del país con tasas del 41,0\%, 38,9\% y $31,1 \%$ respectivamente. A nivel de ocupación todas las ciudades presentan una situación grave con tasas que apenas oscilan entre el $8,8 \%$ y el $13,7 \%$. Además, los niveles de ocupación sin ningún contrato laboral alcanzan preocupantemente tasas del $69,0 \%$ y $81,2 \%$, mientras que la participación de trabajadores que no alcanzan el umbral de 1 SMMLV oscila entre el $61,1 \%$ y 73,6\% de los ocupados; por si fuese poco, las coberturas en capacitación para el trabajo alcanzan un máximo de $6,8 \%$ de PCD atendida a pesar del alto número de requerimientos de capacitación.

Además, la evidencia da indicios de que las ciudades y el país están respondiendo de manera sistemática a las necesidades de inclusión al empleo de la PCD, priorizando las categorías de discapacidad que tienen una mayor representatividad demográfica, lo que está generando, por tanto, disparidades entre la misma PCD, tal caso toma una especial relevancia en la evidencia local de Medellín y Barranquilla; pero es este último caso donde hay mayor presencia de niveles de ocupación más heterogéneos entre los grupos de discapacidad, por lo que la PCD de Barranquilla debe enfrentarse a una mayor desigualdad para emplearse en razón al tipo de discapacidad que presente.

Así mismo, deben priorizarse en la agenda local programas de capacitación laboral para el grupo de PCD de voz y habla, ya que para este tipo de discapacidad las cinco ciudades de análisis presentaron únicamente un total de 78 personas atendidas, además, debe establecerse medidas que orienten a la adaptabilidad de los espacios de trabajo para garantizar la accesibilidad de la PCD de movilidad y de patologías múltiples cuyos grupos presentaron los mayores reclamos de barreras laborales infraestructurales.

Las estimaciones de la función de emparejamiento laboral para PCD muestran altas asimetrías con respecto a las probabilidades de encontrar trabajo y de ocupar vacantes que dejan en evidencia la necesidad de establecer políticas activas de empleo con enfoque local que, entre otros factores, mejoren los procesos y las competencias blandas de búsqueda de empleo para la PCD en Colombia. Por tanto, tales políticas deben diseñarse en contexto con la capacidad local empresarial para generar empleo; con la capacidad local institucional para conectar la demanda y la oferta de trabajo de manera que se reduzcan las asimetrías; $y$, por supuesto, con la capacidad de la gestión local para adaptar medidas que respondan a los requerimientos y atenciones específicas de cada tipo de discapacidad.

Por lo anterior, se hace indispensable replicar un llamado a los hacedores de políticas públicas, para que las medidas de inclusión al mercado laboral se configuren y se adapten desde una perspectiva y tratamiento con enfoque local y descentralizado, que permita, por tanto, la construcción de políticas que respondan con efectividad a las características, problemáticas y oportunidades presentes en el contexto territorial.

No obstante, se encontraron en las ciudades de estudio distintas iniciativas de empleo que consideran el contexto local como una variable clave para la efectividad de las políticas y que presentan buenas prácticas para reducir las asimetrías entre la demanda y la oferta de trabajo, y con ello incrementar la empleabilidad de la PCD, entre los casos locales más exitosos se encontraron el Servicio Público de Empleo, el Programa de Vigilancia Inclusiva Securitas y el Programa Pacto de Productividad.

Tales iniciativas articulan una intervención a nivel poblacional (con procesos de orientación, formación, intermediación, entrega de recursos, vinculación laboral directa, seguimiento post vinculación laboral y participación de mesas locales de cada categoría de discapacidad), a nivel empresarial (con estrategias de acompañamiento, sensibilización, capacitación, diagnóstico, remisión de personas e implementación de ajustes) y a nivel de territorio (con alianza con alcaldías, gobernación y otras entidades de apoyo, consecución de operadores y de clientes locales, análisis de los mercados 
locales de trabajo, procesos de replicabilidad, conformación de redes de trabajo, y diagnósticos y seguimientos territoriales).

Finalmente, se recomienda la importancia de priorizar en la agenda de investigación en discapacidad de Colombia estudios y estrategias de empleo para PCD que se aborden desde un enfoque local, ya que tal perspectiva puede determinar un efecto más directo sobre las problemáticas estructurales del mercado de trabajo al poder realizar una gestión del empleo con base al contexto, dinámicas y ventajas propias del territorio, además de asignar posibilidades de: integrar iniciativas multisectoriales en una estrategia única común al espacio local, trabajar de forma directa en la ordenación de la oferta y la demanda de trabajo, facilitar la coordinación entre los distintos actores (instituciones, empresas, trabajadores) con apropiación por las problemáticas de empleo, atender las necesidades específicas de cualificación del mercado local y apoyarse de las posibilidades potenciales de desarrollo endógeno del territorio.

\section{Agradecimientos}

Esta publicación ha sido posible con el apoyo financiero de la Generalitat Valenciana y de la Cátedra "Aguas de Alicante de Inclusión Social" de la Universidad de Alicante (España). El contenido de esta publicación es responsabilidad exclusiva de los autores y no refleja necesariamente la opinión de la Generalitat Valenciana ni de la Universidad de Alicante.

\section{Referencias}

Agovino, M., \& Rapposelli, A. (2017). Evaluation of the Matching Process of Disabled People Through a Macroeconomic Approach: the Italian Case. Applied Spatial Analysis and Policy, 10(1), 139-159.

Alburquerque, F. (2004). Desarrollo económico local y descentralización en América Latina. Revista de la CEPAL, 82, 157-171.

Antczak, E., Gałecka, E., \& Pater, R. (2018). Unemployment and vacancy flows in spatial labour market matching at the regional level. The case of a transition country. Journal of Applied Economics, 21(1), $25-43$

Berman-Bieler, R. (2007). El desarrollo inclusivo, un aporte universal desde la discapacidad. Washington: Banco Mundial.

Blanchard, O., \& Diamond, P. (1994). Ranking, Unemployment Duration, and Wages. The Review of Economic Studies, 61(3), 417-434.

Cabrero, G., Serrano, C., \& Toharia, L. (2009). Evaluación de las políticas de empleo para personas con discapacidad y formulación y coste económico de nuevas propuestas de integración laboral. Madrid: Cinca.

Castillo, M., Castro, J., Raffo, L., \& Mora, J. (2018). El emparejamiento en el mercado laboral del valle del cauca. Cuadernos de Economía, 37(74), 523-554.

Castro, L. (2017). Análisis sectorial de la función de emparejamiento en Colombia: eficiencia del Servicio Público de Empleo, Revista ESPE - Ensayos sobre Política Económica, 35(83), 101-129.

Coles, M., \& Smith, E. (1996). Cross-Section Estimation of the Matching Function: evidence from England and Wales. Económica, 63(252), 589-597. 
CORONA., ANDI., \& ACDI VOCA. (2016). 15 iniciativas exitosas en materia de inclusión laboral para población vulnerable. Recuperado de http://proyectos.andi.com.co/Gerencia\%20Arquitectura\%20Social\%20-

\%20Fundacion\%20ANDI/Empleo\%20Inclusivo/Documento\%20Interactivo\%20Modelo\%20de\%2 0Empleo.pdf

Correa, L., \& Castro, M. (2016). Disability and Social inclusion in Colombia. Saldarriaga-Concha Foundation Alternative Report to the Committee on the Rights of Persons with Disabilities. Bogotá: Saldarriaga Concha Foundation Press.

Cruz, I., \& Hernández, J. (2010). Magnitud de la discapacidad en Colombia: una aproximación a sus determinantes. Revista Ciencias de la Salud, 6(3), 23-35.

Cruz, I., Duarte, C., Fernández, C., \& García, A. (2017). Hacia la formulación de una Agenda de Investigación en Discapacidad para Colombia. Revista Facultad Nacional de Salud Pública, 35(2), 225-235.

DANE (2018). Reloj de población nacional. Recuperado de: http://www.dane.gov.co/reloj/

Espinosa, O. (2016). Participación laboral de personas en situación de discapacidad. Análisis desde un enfoque de género para Colombia. Economía: teoría y práctica, 45, 137-167.

Fedorets, A., Lottmann, F., \& Stops, M. (2019). Job matching in connected regional and occupational labour markets. Regional Studies, 53(8), 1085-1098.

FOMIM-BID (2015). Reporte final del estado del proyecto ATN/ME-11368-CO: Programa de inserción laboral productiva para personas con discapacidad. Recuperado de https://www.iadb.org/es/project/co-m1033

Fundación Demuca. (2011). Políticas locales para la promoción del empleo. San José, Costa Rica: Demuca.

Hernández, J., \& Hernández, I. (2005). Una aproximación a los costos indirectos de la discapacidad en Colombia. Revista de Salud Pública, 7(2), 130-144.

Infante, B., \& Sunkel, O. (2009). Chile: hacia un desarrollo inclusivo. Revista CEPAL, 97, 135-154.

Jiménez, A., \& Huete, A. (2011). Estudio sobre el agravio comparativo económico que origina la discapacidad. Madrid: Universidad Carlos III de Madrid - Leganés.

McQuaid, R. (2006). Job search success and employability in local labor markets. The Annals of Regional Scienc, 40, 407-421.

Metts, R. (2004). Disability and development. Background paper prepared for the Disability and Development Research Agenda Meeting in the World Bank, November 16, 2004. Washington: World Bank.

Ministerio de Salud y Protección Social (2014). Manual de usuario del aplicativo web Registro para la Localización y Caracterización de Personas con Discapacidad. Bogotá: Gobierno de Colombia.

Ministerio de Salud y Protección Social (2018a). Registro para la Localización y Caracterización de las Personas con Discapacidad del Sistema Integral de Información de la Protección Social. RLCP - SISPRO. Recuperado de: https://reg.discapacidad.sispro.gov.co/RLCPD (junio 30 de 2018). 
Ministerio de Salud y Protección Social (2018b). Sala situacional de las Personas con Discapacidad Febrero 2018, Colombia: Observatorio Nacional de Discapacidad. Recuperado de http://ondiscapacidad.minsalud.gov.co/Paginas/Home.aspx

Ministerio de Trabajo (2018). Servicio Público de Empleo. Recuperado de http://www.serviciodeempleo.gov.co/ (agosto 10 de 2018)

Mora, J., \& Santacruz, J. (2007). Emparejamiento entre desempleados y vacantes para Cali: un análisis con datos de panel. Revista Estudios Gerenciales, 23(105), 85-91.

Mortensen, D. (1986). Job Search and Labor Market Analysis. In Handbook of Labor Economics II, by Richard Layard, Orley Ashenfelter and David Card, 849-919. Amsterdam: Elsevier.

Mortensen, D., \& Pissarides, C. (1999). New Developments in Models of Search in the Labor Market in Handbook of Labor Economics III, by Orley Ashenfelter and David Card, pp. 2567-2627. Amsterdam: Elsevier.

Pacto de Productividad (2015). Pacto de Productividad: Promoviendo la Inclusión Laboral de personas con discapacidad. Bogotá: Puntoaparte.

Parckar, G. (2008). Disability Poverty in the UK. London: Leonard Cheshire Disability.

Pissarides, C. (2000). Equilibrium Unemployment Theory. Second Edition. Cambridge: The MIT Press.

Securitas Colombia (2016). Securitas sostenible: informe Global Reporting Initiative 2016. Recuperado de https://www.securitas.com.co/sostenibilidad/informes-gri/

Securitas Colombia (2018). Empleo sostenible e inclusivo: informe Global Reporting Initiative 2018. Recuperado de https://www.securitas.com.co/sostenibilidad/informes-gri/

Stigler, G. (1962). Information in the Labor Market. Journal of Political Economy, 70(5), 94-105.

Velandia, S., Castillo, M., \& Ramírez, M. (2018). Acceso a la educación superior para personas con discapacidad en Cali, Colombia: paradigmas de pobreza y retos de inclusión. Lecturas de Economía, $89,69-101$. 